\title{
Las segundas generaciones de origen extranjero
}

Este documento se aproxima a la temática de las segundas generaciones de origen inmigrante. En primer lugar, se plantea una reflexión sobre el propio concepto de segunda generación, posteriormente se contextualiza el estudio sobre las segundas generaciones en los estudios migratorios internacionales, españoles y canarios, para luego hacer una somera caracterización de una parte de las segundas generaciones en Canarias, así como contemplar las cuestines sociales más relevantes en torno a esta realidad. Finalmente, se esbozan unas breves conclusiones.

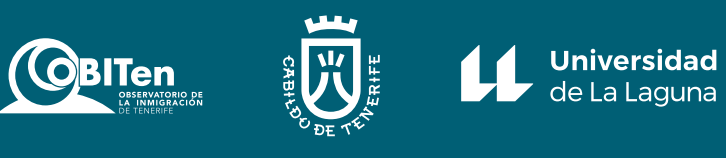




\section{Introducción: ¿mal llamadas segundas generaciones?}

En la literatura sobre migraciones se denomina segunda generación "a los jóvenes nacidos en España de padres extranjeros o traídos al país antes de los 12 años" (Portes y Aparicio, 2013, p. 1). También se conoce como generación 1.5 a los niños que vienen con sus padres a una edad temprana y experimentan la mayor parte de su socialización primaria en la sociedad de asentamiento (Aparicio, 2007). En los estudios sobre hijos e hijas de personas inmigrantes se ha consolidado el término de segunda generación para referirse a éstos. Sin embargo, estas personas nunca migraron, aunque en muchos casos mantienen la nacionalidad extranjera heredada de sus progenitores.

Es necesario señalar la diferencia entre segunda generación de origen inmigrante y jóvenes inmigrantes. La primera categoría hace referencia a una cohorte generacional descendiente de personas que han inmigrado en una sociedad diferente a su sociedad de origen. Por tanto, la segunda generación serían sus descendientes que o bien han nacido en la sociedad de destino de sus progenitores, o han llegado a ella en su infancia y han experimentado parte de su socialización en la misma. Por otro lado, hacemos referencia a jóvenes inmigrantes cuando éstos se han desplazado de un lugar de origen a otro de destino siendo mayores de 12 años, por tanto, sería una categoría social que equivale a un intervalo de edad determinado dentro de un colectivo poblacional epecífico.

En algunos estudios se plantea que la denominación de segunda generación de inmigrantes funciona para generar una frontera simbólica entre el grupo de inmigrantes y sus hijos, por un lado y los autóctonos, por otro. Es decir, "se construye sobre una clasificación que equipara a los hijos de inmigrantes con sus padres, y los opone a los autóctonos" (García Borrego, 2003, p. 2). Tampoco podemos obviar, en este sentido, la carga normalmente peyorativa que implica el término inmigrante y que, por tanto, también tiene una función estigmatizante para quiénes son incluidos en el mismo colectivo. En este sentido, la reflexión que cabría hacer es ¿cuándo un inmigrante deja de serlo? ¿por qué se identifica a los hijos de inmigrantes como "hijos de"? Según García Borrego (2003), caracterizar a una población a partir de su filiación está próximo a una forma de racismo culturalista.

Por otro lado, Aparicio y Tornes $(2007$, p. 23) proponen que tal concepto no sea entendido en términos biológicos sino histórico-políticos. En términos históricos dado que son segundas generaciones temporalmente diferenciadas con respecto a sectores de la población anteriores a ellas y, por tanto, enfrentan situaciones distintas que deben ser comprehendidas. Políticamente porque si no se tuvieran en cuenta desde ese ámbito, "se desatendería a lo generacionalmente específico de las actuales minorías descendientes de inmigrados".

Más alla de su conceptualización, simbólicamente importante y sociológicamente necesaria, hay múltiples temas relacionados con las generaciones culturalmente diversas que residen en la sociedad canaria en general y tinerfeña en particular que deben ser puestos de relieve para entender y atender la situación actual de nuestras sociedades. ¿Qué se ha estudiado sobre ellas? ¿Cuáles son sus características sociodemográficas? ¿Qué otras cuestiones sociales están detrás de estas realidades? o ¿Cómo se está gestionando la diversidad cultural en la sociedad y en las instituciones educativas actualmente? A todas estas preguntas trataremos de responder en los siguientes apartados. 


\section{El estudio de las segundas generaciones}

Los estudios sobre segundas generaciones comienzan a proliferar en España en la primera década del siglo XXI. Sin embargo, no es un campo muy explorado y aun se encuentra poca bibliografía empírica al respecto, aunque no es de extrañar teniendo en cuenta que España se convierte en un país de inmigración de manera más tardía que otros, donde dichos estudios comienzan antes, tales como Estados Unidos u otros países europeos. En Estados Unidos es a partir de la década de los 50 y especialmente de los 60 que se ponen de manifiesto las fracturas sociales existentes por cuestiones raciales y se comienza a cuestionar el papel de la escuela como melting pot (Aparicio, 2007). En algunos países europeos como Alemania o Francia se comienza a notar la preocupación por la integración de las segundas generaciones de origen extranjero cuando se experimentan aontecimientos que ponen en riesgo las perspectivas de convivencia pacífica y seguridad ciudadana.

Por tanto, los estudios sobre las segundas generaciones se estrenan en Estados Unidos, para después comenzar en Europa en la década de los 90 y en España en la primera década del nuevo milenio. Además, los estudios norteamericanos y europeos responden a preocupaciones diferentes. En Estados Unidos se basan en el incumplimiento de las expectativas de futuro del país (el sueño americano), mientras que en Europa tienen que ver con cuestiones más "inmediatas e individualistas (amenazas para la seguridad ciudadana y la paz social) o bien en criterios un tanto elitistas (la quiebra de los derechos humanos y del modelo social europeo que se supone subyacer a los deficientes niveles de integración)" (Aparicio y Tornes, 2007, p. 30). Sin embargo, ambos enfoques acaban confluyendo en la importancia dada a los logros escolares y laborales de la segunda generación como condicionantes de su grado de integración.

La teoría de la asimilación segmentada es uno de los enfoques empleados actualmente en la aproximación a esta realidad. Esta teoría vino a sustituir las teorías clásicas de la asimilación desde las que se concibe como una tendencia inevitable que el paso del tiempo termine produciendo la asimilación por la sociedad de acogida, según la tesis sobre el ciclo de relaciones interétnicas (Park, 1930). La teoría de la asimilación segmentada, frente a la teoría clásica de la asimilación, afirma que los hijos de los inmigrantes van a seguir trayectorias diferenciadas (mayor o menor éxito social) y que ello dependerá de los recursos (capital social, económico o cultural) de los que dispongan tanto ellos como sus familias. Esta teoría también contempla la importancia del contexto (percepción del grupo etnonacional en la sociedad receptora) y las relaciones intergeneracionales.

Entre los estudios europeos más relevantes cabe destacar el llamado Effectiveness of National Policies in the Integration of Children of Immigrants (EFFNATIS) ${ }^{1}$, publicado en 2001, que consistió en estudiar las políticas de integración en una serie de países europeos (Francia, Alemania y Gran Bretaña) y las consecuencias que éstas tenían sobre la integración de los hijos e hijas de las personas inmigrantes. Sus principales conclusiones se pueden resumir en las siguientes: primero, que ninguno de los tres modelos nacionales de integración (el asimilacionista francés, el multiculturlista británico o el de la comunidad étnica alemán) son eficaces en la consecución de sus objetivos; segundo, que son más efectivas las políticas generales aplicadas a toda la población que las específicas dirigidas a un colectivo determinado; y tercero, que los logros educativos y laborales de los hijos e hijas de inmigrantes eran menores que los alcanzados por la población autóctona.

1 Más información sobre el proyecto y acceso al informe final disponible en http://www.efms.uni-bamberg.de/ prineffe.htm. 
Otro estudio comparativo más reciente ha sido el estudio europeo Reducing School Leaving in the European Union ${ }^{2}$ en nueve países comunitarios con diferentes indicadores de abandono temprano de educación y formación (ATEF) centrado en el papel que en este proceso juegan las aspiraciones y la percepción del apoyo familiar y docente, concluyendo en muchos casos la coexistecia de altas aspiraciones con prácticas excluyentes por parte del profesorado. En el caso español, el estudio mostró una de las más altas tasas de ATEF en el marco de los países de la UE-28 con mayor brecha por género y país de nacimiento o nacionalidad (Carrasco, Pàmies y Narciso, 2018).

En el contexto español disponemos del estudio más importante a nivel europeo en términos de tamaño muestral sobre segundas generaciones de origen extranjero y uno de los primeros realizados sobre esta temática a nivel estatal. Se trata de la Investigación Longitudinal sobre la Segunda Generación en España (ILSEG) que se llevó a cabo en tres momentos diferentes entre los años 2006 y $2017^{3}$. El estudio empleó una metodología cuantitativa con encuestas tanto a población de origen extranjero como nativo empleando para su análisis el enfoque de la teoría de la asimilación segmentada. Se trabajó sobre diferentes variables, unas de tipo estructural: sociodemográficas (edad, estado civil, tipo de residencia); educación, aspiraciones educacionales y lenguaje; empleo, estatus ocupacional e ingresos; y otras más de tipo subjetivo: percepciones de discriminación y autoidentificación nacional.

Los resultados muestran similitudes entre los hijos de inmigrantes y de nativos en las variables de la ambición de futuro, las percepcioes de discriminación y la autoidentidad nacional común. De manera que el estudio concluye con un balance positivo del proceso de integración de la segunda generación en España. Sin embargo, se encuentran factores de desventaja para el grupo desdenciente de inmigrantes, tales como la desventaja económica, la mayor dificultad para acceder a niveles ocupacionales más altos y el efecto de la religión musulmana en las experiencias de discriminación (Portes, Aparicio y Haller, 2018, p. 166).

Del estudio citado anteriormente - ILSEG - se extrajo una submuestra de los hijos e hijas de personas inmigrantes y autóctonas ya incorporadas al mercado laboral que, junto con otras aproximaciones cualitativas, dieron lugar a otra publicación sobre la inserción laboral de las segundas generaciones al mercado laboral español y sus experiencias de discriminación. Este informe concluye que el colectivo de origen inmigrante estaría en desventaja con respecto a sus homólogos de origen nativo en los puestos ocupados en el mercado laboral. Este hecho "parecería apuntar a la existencia de un cierto nivel de discriminación por parte de las empresas a la hora de seleccionar y de contratar jóvenes de origen inmigrante" (Aparicio, Biderbost y Tornos, 2019, p. 109).

En el contexto canario no existen estudios sobre segundas generaciones como objeto de etsudio, sino que estas quedarían subsumidas en aproximaciones hacia los jóvenes inmigrantes. Teniendo esto en cuenta, existen algunos artículos referidos al caso canario sobre el perfil sociodemográfico de los jóvenes de nacionalidad extranjera (Domingo y Bayona, 2007); buenas prácticas de integración, tomando como ejemplo el proyecto europeo INVOLVE donde participó como invitado el Observatorio de la Inmigración de Tenerife (Fariña Álvarez y Obiten, 2007); y sobre inmigración, juventudes y planificación social en Canarias (Kuehn Dumpiérrez, 2007).

2 Más información del proyecto disponible en http://sprc.info/reducing-early-school-leaving-in-europe/.

3 Los resultados se han recogido en diferentes publicaciones: Aparicio (2007); Portes, Aparicio y Haller (2009); Portes y Aparicio (2013); y Portes, Aparicio y Haller (2018). 
Otro de los trabajos realizados en Canarias es el informe sobre Inmigración, Escolarización y Educación Intercultural en Canarias (2007) donde se abordan las características sociales y académicas del alumnado de origen inmigrante, sin diferenciar a las segundas generaciones, en los diferentes niveles educativos preuniversitarios. Entre sus conclusiones y de manera muy sintética cabe destacar el mayor nivel de absentismo escolar entre el alumnado de origen inmigrante con respecto al autóctono; el bajo nivel de conflictividad; la menor implicación en actividades extraescolares del colectivo de origen inmigrante; los escasos servicios de apoyo en algunos municipios; el bajo nivel de dificultades escolares $y$, por tanto, alto nivel de integración.

Por último, y siendo la única publicación referida exclusivamente a los hijos e hijas de personas inmigrantes, en un artículo de la Revista Española de Sociología se analiza su rendimiento académico en Canarias con datos de PISA y que, en palabras de los autores, es un "caso obviado por la literatura y única comunidad en la que este alumnado no presenta una desventaja académica" en comparación a otros países europeos y al resto del estado español (Álvarez-Sotomayor, Gutiérres Rubio y Martínez-Cousinou, 2018, p. 83). En el artículo se concluye que Canarias es la única comunidad autónoma española donde los hijos de inmigrantes tienen semejantes puntuaciones a los nativos en los datos de PISA, no porque los primeros tengan mejores resultados que sus homólogos en otras regiones pues, al contrario, es bastante similar, sino por el peor rendimiento relativo del alumado de origen canario, por debajo de las puntuaciones del resto de comunidades. En lo único que no se evidencian tantas diferencias es que los resultados académicos de los hijos de inmigrantes son mejores que los de su generación precedente, aunque estas diferencias también son menores en comparación con el resto del país.

\section{La caracterización de las segundas generaciones en Canarias}

Las segundas generaciones en Canarias no son un fenómeno reciente, ya existían hijos de inmigrantes comunitarios, noruegos y suizos, principalmente; pero también asiáticos, entre ellos, indios, que comienzan a instalarse desde finales del siglo XIX; coreanos, que se asientan en torno a la actividad portuaria de las Islas en torno a los años 60; y procedentes de Oriente Medio (palestinos, sirios, libaneses), que comienzan a llegar en la primera mitad del siglo XX, así como población mauritana y originaria del Sahara Occidental (relacionados con el comercio de maleta). Sin embargo, las segundas generaciones son más bien un fenómeno que se ha visibilizado recientemente dado el impacto mediático que se le ha dado, sobre todo, a los menores extranjeros no acompañados. Por otra parte, lo que sí ha cambiado son las nacionalidades de procedencia y su peso sobre el total de población extranjera o de origen extranjero que, evidentemente, va en aumento.

Una de las principales dificultades en el estudio de las segundas generaciones de origen extranjero es la falta de datos estadísticos adecuados ya que, por ejemplo, los datos disponibles no permiten identificar a las segundas generaciones. Por tanto, para aproximarnos a la caracterización de los hijos e hijas de las personas inmigrantes, hemos tomado como fuente de datos el número de nacimientos en Canarias según nacionalidad de las madres y los padres ${ }^{4}$. Sin embargo, estos gráficos hay que tomarlos con cierta precaución ya que tampoco recogen totalmente la realidad de las segundas generaciones en Canarias, puesto que en ellos no se recogen ni las personas llegadas a Canarias en su niñez, ni los movimientos de la población (personas nacidas en Canarias que se hayan ido o desplazado a otro lugar diferente a la isla de nacimiento), ni tampoco los nacidos de madres o padres de origen extranjero pero nacionalizados.

4 Datos del ISTAC. 


\section{Gráfico 1}

Evolución de

nacimientos en Canarias

según nacionalidad

extranjera de las madres

y los padres.

Fuente

ISTAC. Natalidad, Movimiento natural.

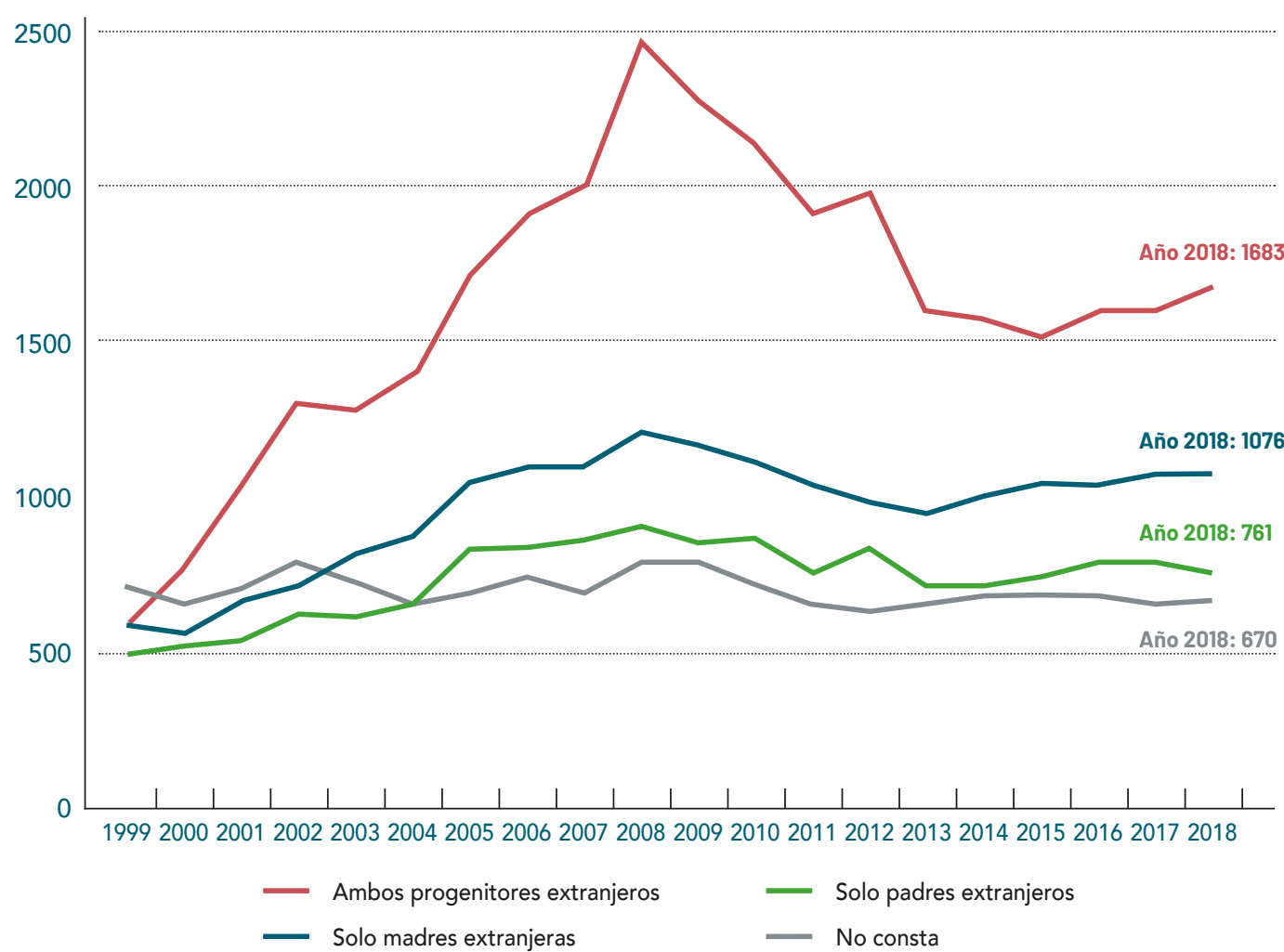

Como se puede observar en el Gráfico $1^{5}$ sobre la evolución de nacimientos en Canarias de madres y/o padres extranjeros, el mayor número de nacimientos se produce de progenitores extranjeros, tanto la madre como el padre. En segundo lugar, estarían los nacimientos en los que solo la madre es extranjera, sin embargo, aquí no disponemos de información para saber la nacionalidad del padre o incluso la existencia del mismo, ya que pueden ser madres solteras, o el progenitor puede ser nacional o no estar en Canarias en el momento del nacimiento. En tercer lugar, estarían los nacmientos cuyo padre es el único extranjero, ententiendo, por tanto, que la madre tiene nacionalidad española (no sabemos si es autóctona o nacionalizada). En cualquier caso, se ha querido reflejar tanto a los hijos e hijas de madres y padres extranjeros como a los descendientes de parejas culturalmente mixtas, aunque tampoco disponemos de datos para conocer exactamente las características de los progenitores. En los tres casos, el año de mayor nacimientos tuvo lugar en 2008, con 2.460 nacimientos con ambos rogenitores extranjeros, 1.213 nacidos de solo madres extranjeras, y 907 nacimientos donde solo el padre es extranjero. A partir de dicho año se produce un descenso en los nacimientos con algún repunte en el caso de los nacimientos de ambos progenitores extranjeros y solo padres extranjeros en el año 2012, produciéndose un ligero ascenso a partir del año 2015 que se ha sostenido hasta 2018, salvo en el caso de los nacimientos de solo padres extranjeros que cayó en 2018 con respecto a 2017.

En el Gráfico 2 se refleja la proporción de nacimientos de madres y/o padres extranjeros frente a los nacimientos de progenitores con nacionalidad española. Como se puede observar, el número de nacimientos de origen extranjero va en ascenso con los años, llegando a su máximo en 2008, año a partir del que se produce un descenso que revierte a partir del año 2013 hasta el último año del que se disponen datos. Si bien la proporción de nacimientos de origen extranjero en Canarias en el año 1999 era del 8,9\% con respecto al

5 Falta de información de los Registros Civiles de los municipios de Antigua (2004), Puerto del Rosario (2007, 2004), Yaiza (2004) y Arona (2003 y 2004). Esto se aplica también a los gráficos 3, 5 y 6. 


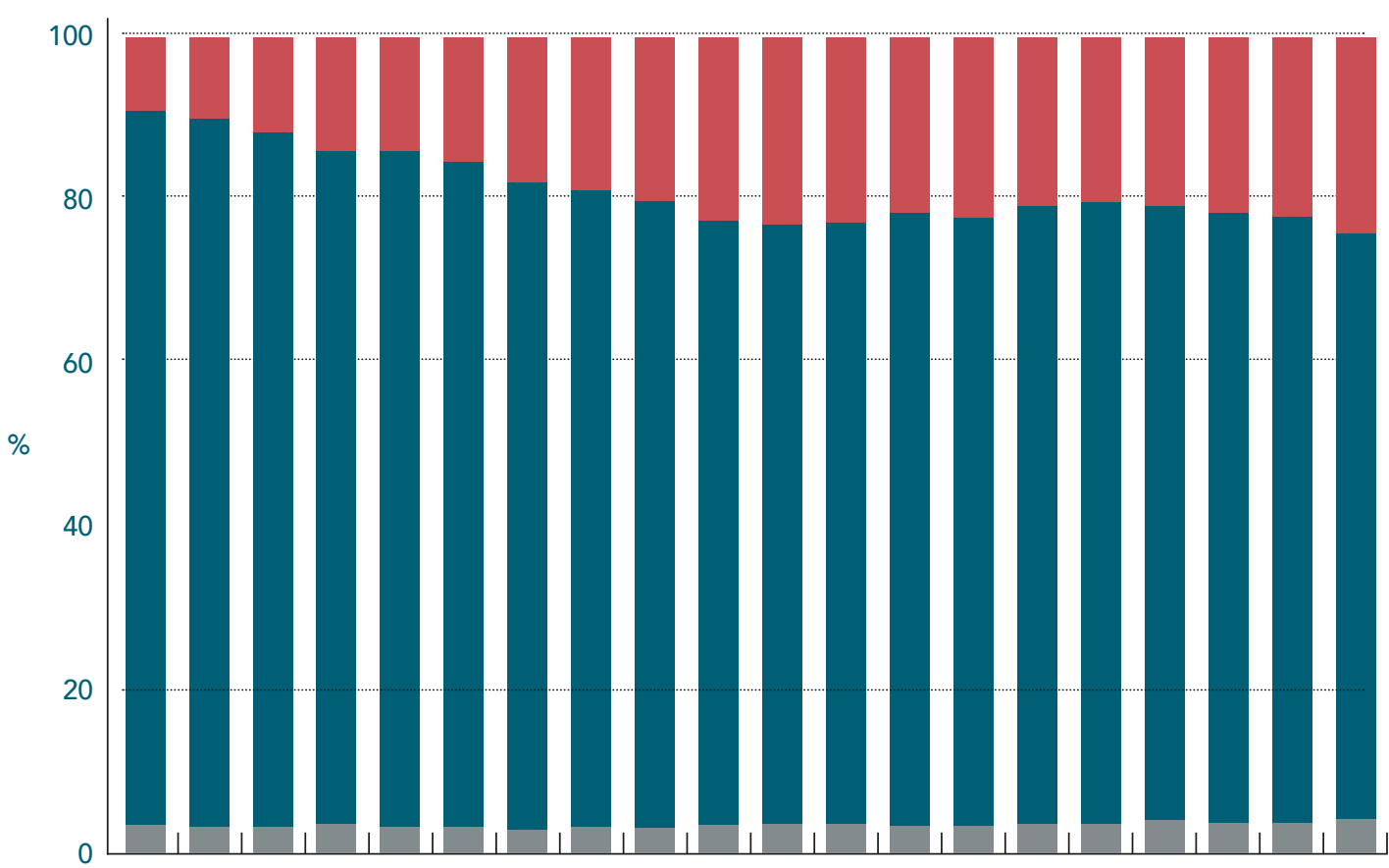

19992000200120022003200420052006200720082009201020112012201320142015201620172018

Ambos o algún progenitor extranjero

Ambos progenitores españoles

\section{Gráfico 2}

Proporción de

nacimientos en Canarias según nacionalidad de las madres y los padres.

\section{Fuente}

ISTAC. Natalidad,

Movimiento natural.

total de nacimientos, la mayor proporción se alcanza en el año 2009 con un 22,7\% y esto tiene que ver no solo con el mayor número de nacimientos de origen extranjero sino con un menor número de nacimientos de nacionales. Sin embargo, es en el año 2018 cuando se alcanza la mayor proporción, alcanzando el $23,5 \%$ de los nacimientos totales, y que está relacionado con el motivo mencionado anteriormente.

En la siguiente figura, Gráfico 3, se contempla la poporción del número total de nacimiento desde 1999 hasta 2018 en comparación con la población con nacionalidad española nacida en esos años en Canarias. El gráfico responde a la pregunta sobre cuál es la proporción de población menor de 20 años nacida en Canarias y de origen extranjero con respecto a la nacional. Tal y como se muestra en el gráfico, el 77,9\% de la población nacida en Canarias de madres y/o padres españoles tiene menos de 20 años, un 9,3\% ha nacido en el seno de familias con algún progenitor extanjero, y el 8,9\% con ambos progenitores extranjeros.

En general para el periodo estudiado (1999-2018), la isla que mayor número de nacimientos registra es Tenerife (con 1.500 nacimientos), con la excepción de los años 2003 y 2004 donde se registraron más en Gran Canaria. Por tanto, Gran Canaria sería la segunda en orden descendente, con 948 nacimientos en 2018, seguida por Lanzarote (515), Fuerteventura (428), La Palma (91), La Gomera (23) y El Hierro (15). Si bien en Tenerife, y en el resto de las islas, no se han alcanzado

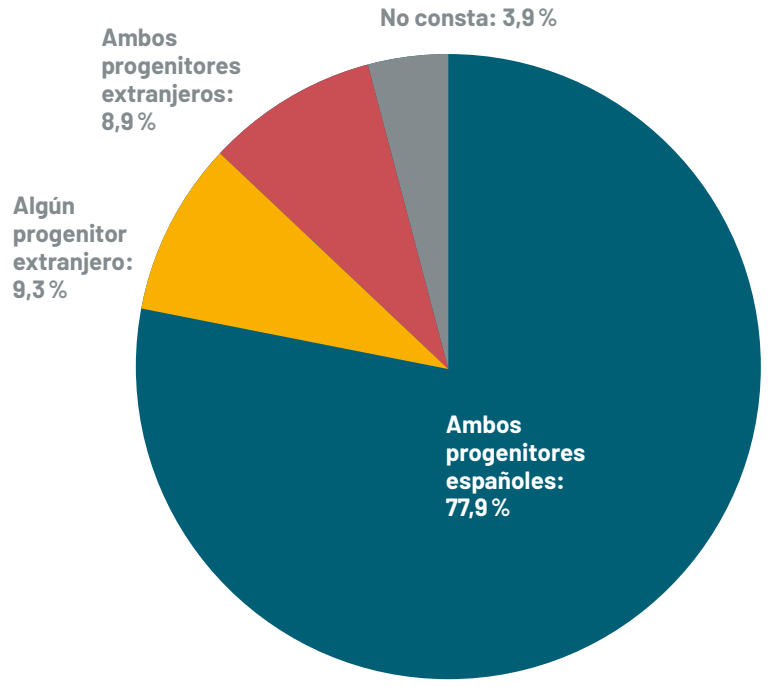

\section{Gráfico 3}

Proporción de personas menores de 20 años según nacionalidad de las madres (Año 2018).

\section{Fuente}

ISTAC. Natalidad, Movimiento natural. 
las cifras de nacimientos del año 2008, es donde, junto a Lanzarote y La Palma donde los nacimientos han aumentado en 2018 con respecto al año anterior, en el resto (Gran Canaria y Fuerteventura) han descendido, mientras que en El Hierro y La Gomera se mantienen estables.

En cuanto a los orígenes de procedencia, la mayoría de nacimientos se registra de madres procedentes de América, el segundo mayor volumen de nacimientos se produce entre madres de origen europeo. Los nacimientos de madres africanas, en tercer lugar, han mantenido una evolución con altibajos con tendencia descendente en los últimos años. Por último, y con menor número de nacimientos se encuentran las madres de origen asiático con una evolución bastante estable y con una tendencia a la baja pero de manera muy poco significativa desde el año 2014.

\section{Otras cuestiones relevantes sobre las segundas generaciones}

La aproximación a las segundas generaciones de origen inmigrante se suele hacer desde el estudio de su integración: qué nivel de estudios ha adquirido y qué tipo de ocupación tiene, todo esto en términos comparativos a su generación precedente para concluir la dirección de dicha integración, es decir, si reproduce el mismo patrón que sus padres, lo supera con un mayor éxito educativo y/o laboral o, por el contrario, se encamina hacia la exclusión social.

Sin embargo, hay otras cuestiones no menos importantes que incluso pueden estar relacionadas con la posición social, pero que tienen que ver más con las percepciones, identificaciones y experiencias que viven las personas de segunda generación de origen inmigrante. A su vez, las percepciones, identificaciones y experiencias pueden estar relacionadas entre sí de distinto modo, tal y como veremos a continuación.

Entre la segunda generación se observa la denominada paradoja de la integración social (Heath, 2014, p. 8). Esto es, a mayor integración social, mayor consciencia poseen de la discriminación que enfrentan. Esto es así porque sus expectativas de aceptación e integración en la sociedad de residencia son mayores y, en no pocas ocasiones, se ven frustradas por la visibilización de las diferencias respecto al grupo mayoritario (marcadores identitarios o culturales como los rasgos fenotípicos, vestimenta e idioma). La discriminación en la sociedad receptora influye negativamente en la identificación de los sujetos con su lugar de residencia.

Por tanto, la pregunta que aquí cabe hacerse es hasta qué punto el origen de las segundas generaciones condiciona sus experiencias vitales en lo que éstas consideran su propia sociedad al haber nacido en ella. La hipótesis que cabría plantear es que probablemente mientras más se visibilizan los marcadores identitarios, entendiendo estos como elementos que señalan la diferencia de estas personas con respecto al grupo autóctono, mayor serán las experiencias de discriminación de las mismas en tanto que serán percibidas como "inmigrantes" sin realmente serlo.

Estas experiencias pueden tener consecuencias que implican, en cierto modo, una profecía que se cumple a sí misma, ya que el hecho de que determinados colectivos culturalmente diferenciados se perciban fuera de las propias fronteras grupales, generará una mayor percepción de distanciamiento social en aquéllos. 
En este sentido, algunos estudios han investigado sobre las causas que propician el aislamiento social de estas segundas generaciones. Es el caso del artículo sobre la segunda generación de paquistaníes británicos en el Reino Unido (Khan, 2011, p. 67). En concreto, el autor se pregunta si la observada identificación de éstos con el lugar de origen de sus familias viene dada por la hostilidad de la sociedad británica hacia la población musulmana o si, por el contrario, es su resistencia a la asimilación lo que ha generado esta distancia y hostilidad con la sociedad local. En esta misma línea, el autor emplea el término "etnicidad reactiva" de Ruben G. Rumbaut (2008), que ilustra cómo los individuos "expuestos al mensaje de que, por razones raciales o culturales, no forman parte de la corriente social dominante" (Portes, Vickstrom y Aparicio, 2013, p. 231), suelen replegarse en sus identidades étnicas (Rumbaut, 2008).

Hay otra cuestión importante en este ámbito y son los discursos de la población autóctona con respecto a las minorías étnicas donde se incluyen las segundas generaciones diversas culturalmente. Discursos que, a su vez, están directamente relacionados con las percepciones y experiencias de las personas de origen inmigrante en relación a lo comentado enteriormente. En esta dimensión, cobra especial importancia la islamofobia, y en concreto la islamofobia de género, ya que es en la imagen de las mujeres y de las jóvenes con pañuelo donde se concentran buena parte de los fenómenos islamófobos (Ramírez y Mijares, 2005). De este modo, algunos estudios apuntan una mayor discriminación hacia las mujeres musulmanas con hiyab dada la mayor visibilidad de una identidad cultural estigmatizada (Aixelá, 2001). En el caso concreto de las mujeres musulmanas de segunda generación, éstas son percibidas desde un enfoque etnocentrista que las despoja de su valor social (Goffman, 1986) y, por consiguiente, de su condición de ciudadananas, no siendo reconocidas como iguales. En no pocas ocaciones estos discursos de hostilidad hacia ellas se legitiman en valores democráticos tales como la igualdad de género, teóricamente opuestos al hecho de "velarse", lo que las convierte en objetos del llamado racismo democrático (Buraschi y Aguilar Idáñez, 2019). Sin embargo, el peligro de estos fenómenos estriba precisamente en las consecuencias que tiene la discriminación y aislamiento social de estas mujeres al relegarlas a situaciones de dicriminación en el acceso al mercado laboral, entre otras.

Las diferencias pueden ser mantenidas por el rechazo a la asimilación, en particular, cuando la visibilización de las diferencias raciales y religiosas, suscitan la discriminación y la exclusión (Tölölyan, 2007). Estas fronteras (o diferencias) étnicas son simbólicas. Esto es, "distinciones conceptuales hechas por los actores sociales para categorizar objetos, gente, prácticas e incluso tiempo y espacio" (Lamont y Molnár, 2002, p. 168); y deben ser diferenciadas de las fronteras sociales, que "son formas materializadas de diferencias sociales manifestadas en un desigual acceso y desigual distribución de recursos (materiales e inmateriales) y oportunidades sociales" (Lamont y Molnár, 2002, p. 168). Aquí la pregunta sería de qué modo las fronteras simbólicas pueden conllevar al establecimiento de fronteras sociales.

En general y desde el punto de vista de las teorías clásicas de la asimilación, se espera que a más tiempo de residencia, el grado de integración y ascenso social sean más elevados; y que la segunda generación se sienta más identificada y esté mejor integrada con su sociedad de residencia que la primera generación. Pero la estructura local de oportunidades condiciona la adaptación de estas generaciones a sus respectivos contextos sociales, junto a otros factores. Si esta estructura no facilita la integración de los sujetos se intensifica la separación entre el grupo minoritario y el mayoritario (Esman, 2009, p. 100).

Por tanto, y acorde con la dimensión bidireccional del proceso de integración, es fundamental generar no solo un cambio en el imaginario de la composición cultural de las 
sociedades actuales, sino también en la necesidad de diseñar políticas públicas que contribuyan a integrar a los diferentes grupos culturales y corregir los diferentes tipos de racismo que se despliegan en las sociedades receptoras de inmigración.

En cuanto a la planificación social autonómica relativa a la inmigración se materializa por primera vez en Canarias a través del Plan Canario para la Inmigración aprobado en 2002. Sin embargo, este Plan no estableció ni una conceptualización de la integración ni un modelo a seguir, dada la dificultad de alcanzar un consenso político y administrativo para ello en aquel momento (Kuehn, 2007). Cabe señalar la existencia del Foro Canario de la Inmigración, creado en el año 2000, y que se constituyó como un órgano de participación, consulta y asesoramiento del Gobierno de Canarias y que reúne a agentes políticos y sociales clave en dicha materia, aún en vigor.

En el mismo año 2002 se firmó el Pacto Canario sobre Inmigración por varias fuerzas políticas donde se adoptaban varios acuerdos en torno a la inmigración, básicamente en torno a la necesidad de controlar la llegada de inmigrantes por vía irregular, velar por el cumplimiento de sus derechos y fomentar valores interculturales en la sociedad canaria. Sin embargo, el pacto pone mayor énfasis en el primer elemento: la llegada de inmigración y su acogida.

Al primer Plan le siguió un segundo, el II Plan para la Inmigración 2006-2008, donde se mencionaba por primera vez a los jóvenes inmigrantes más allá de los menores extranjeros no acompañados y que suponía un paso más por cuanto se intentó trascender la acción exclsuivamente asistencial del primero así como se establecía también la necesaria sensibilización de la sociedad de acogida como agente clave en la integración de la población extranjera y la diversidad cultural.

\section{A modo de conclusión}

Tomando los datos aquí recogidos sobre nacimientos en Canarias para el año 2018, podemos concluir que, estimativamente, un $18,2 \%$ de los nacimientos producicos ese año se han producido en parejas extranjeras o parejas mixtas (con uno de los progenitores con nacionalidad extranjera). El 25,5\% de las personas nacidas desde 1999 en Canarias de origen extranjero (con dos o uno de sus progenitores con nacionalidad etxranjera) tiene actualmente entre 0 y 4 años; el 28,2\% está entre 5 y 9 años; el $28 \%$ entre 10 y 14 años; y el $18,3 \%$ entre 15 y 19 años.

Esto responde a que los años que mayor número de nacimientos registraron fueron el 2008 y los años inmediatamente posteriores. Si bien en el año 2008 el mayor número de nacimientos era de madres de origen americano, probablemente de los países lationamercanos más representados en Canarias, en el año 2018 las mayores cifras de nacimientos son de origen europeo comunitario, en coherencia con la mayor presencia de este colectivo en el Archipiélago, siendo el origen americano el que se encuentra en segundo lugar, seguido del africano y el asiático. Acorde con el mayor tamaño de la población extranjera, las islas que registran más nacimientos son Tenerife y Gran Canaria.

En síntesis, podemos afirmar que el estudio de las segundas generaciones se hace normalmente en términos comparativos con el de su generación precedente con el fin de analizar la evolución en su proceso de integración en la sociedad de acogida. De este modo, las publicaciones que abordan esta temática se suelen plantear desde una perspectiva socioes- 
tructural, esto es, analizando su posición social. Hay otro tipo de trabajos que también se centran en las cuestiones identitarias y la discriminación experimentada por los colectivos de origen extranjero.

Sin embargo, aun escasean los estudios empíricos en España en general y en Canarias yTenerife, en particular. Es cierto que aun deberán pasar algunas décadas para poder observar las trayectorias por completo (cuando estos jóvenes se inserten en el mercado laboral) pero ya existe un porcentaje de población de origen extranjero que puede darnos mucha información sobre cómo se están integrando estas generaciones en el sistema educativo; qué resultados están obteniendo; si viven experiencias de discriminación; cómo se autoidentifican; e incluso estudios con perspectiva interseccional de género, clase y etnia entre otras cuestiones. Como hemos comprobado en el análisis del caso canario con los datos de PISA, en Canarias parece darse una integración a la baja ${ }^{6}$ de la población inmigrante (Álvarez-Sotomayor, Gutiérres Rubio y Martínez-Cousinou, 2018).

En materia educativa, por ejemplo, la LOE recoge la intervención con el alumnado de origen extranjero mediante el apoyo idiomático, las aulas de acogida y la educación intercultural. Sin embargo, la educación intercultural no suele integrarse en el currículum escolar, sino forma parte de las acciones puntuales que se deja a voluntad de los centros educativos y donde muchas veces se exotiza la diferencia cultural mediante las celebraciones "del día de..." como algo excepcional y no como un aspecto que convive en el marco de nuestro propio entorno social.

En este sentido, es necesario generar datos sobre la integración de las segundas generaciones en términos comparativos con los de sus generaciones precedentes para poder comprender su evolución, tanto educativos como laborales. Sin embargo, no habría que centrarse únicamente en estudios esructurales, que analicen las posiciones sociales, sino también que den cuenta de otras cuestiones relevantes que han sido abordadas en este documento, tales como las experiencias de discriminación, empleando un enfoque interseccional. Esto es, en función del origen, la clase social y el sexo, así como de los marcadores identitarios (en especial de los rasgos fenotípicos y la vestimenta), y teniendo en cuenta también los espacios donde se producen así como las consecuencias que dichas experiencias conllevan; las cuestiones identitarias, es decir, que se aborden las identificaciones de las segundas generaciones con respecto a sus diferentes grupos de pertenencia y referencia; las expectativas; $y$ en general las narrativas de estas personas acerca de sus experiencias vitales que nos ayuden a identificar los mecanismos de integración y exclusión que se despliegan en el actual contexto de la sociedad canaria.

Es necesario tener información al respecto para orientar políticas públicas que hasta ahora no se han materializado en acciones concretas donde la diversidad cultural sea enfocada como un elemento constitutivo de la propia sociedad, ya que cada vez más la población canaria ha nacido en familias de origen extranjero. Las políticas públicas no deberían dejar de lado estas cuestiones teniendo en cuenta los negativos resultados que pueden tener las experiencias de discriminación o exclusión social (véase el ejempleo francés) y, sobre todo, que el racismo y la discriminación son los principales obstáculos que afrontan los inmigrantes y sus familias en las sociedades de destino. Sociedades que son consideradas por sus descendientes como propias al haber nacido en ellas y cuyas expectativas de integración, mayores que las de sus progenitores, pueden verse truncadas por experiencias de discriminación.

6 Para profundizar en un análisis actualizado acerca de las condiciones socioeconómicas de la población en Canarias, véase el estudio sobre Migraciones, desigualdad e integración en tiempos de crisis de Godenau y Buraschi (2017). 
Para ampliar la información

AIXELÁ CABRÉ, Y. (2001). "¿Qué nos ofende de los 'moros'? Discurso sobre los musulmanes y sus prácticas sociales", Scripta Nova. Revista Electrónica de Geografía y Ciencias Sociales, 94(59).

ÁlVAREZ-SOTOMAYOR, A., GUTIÉRRES RUBIO, D. y MARTÍNEZ-COUSINOU, G. (2018). ¿Desventaja académica de los hijos de inmigrantes? La excepción canaria en el contexto español. Revista Española de Sociología, 27(1), pp. 83-106.

APARICIO GÓMEZ, R. (2007). Las segundas generaciones en España: marroquíes, dominicanos y peruanos. Cuadernos Europeos de Deusto, 36, pp. 19-56.

APARICIO GÓMEZ, R. y TORNOS CUBILLO, A. (2007). Hijos de inmigrantes que se hacen adultos: marroquíes, dominicanos y peruanos. Ministerio de Trabajo y Asuntos Sociales.

BURASCHI, D. y AGUILAR IDÁÑEZ, M.J. (2019). Racismo y antirracismo. Comprender para transformar. Universidad de Castilla-La Mancha.

CACHÓN RODRÍGUEZ, L. y LÓPEZ SALA, A. (Coords.) (2007). Juventud e Inmigración, Desafíos para la Participación y para la Integración. Dirección General de Juventud de la Consejería de Empleo y Asuntos Sociales del Gobierno de Canarias.

CARRASCO, S., PÀMIES, J. y NARCISO, L. (2018). Abandono escolar prematuro y alumnado de origen extranjero en España ¿un problema invisible? Anuario CIDOB de la Inmigración 2018, pp. 212-236.

DOMINGO, A. Y BAYONA, J. (2007). Perfil sociodemográfico de los jóvenes de nacionalidad extranjera en España y las Islas Canarias. En Cachón Rodríguez, L. y López Sala, A. (Coords.) (2007). Juventud e Inmigración. Desafíos para la Participación y para la Integración, pp. 12-26. Dirección General de Juventud de la Consejería de Empleo y Asuntos Sociales del Gobierno de Canarias.

ESMAN, M. J. (2009). Diasporas in the Contemporary World. Cambridge: Polity.

FARIÑA ÁlVAREZ, J. Y OBITEN (2007). Proyecto Involve. Participación de inmigrantes en actividades de voluntariado para mejorar la integración. En Cachón Rodríguez, L. y López Sala, A. (Coords.) (2007). Juventud e Inmigración, Desafíos para la Participación y para la Integración, pp. 260-270. Dirección General de Juventud de la Consejería de Empleo y Asuntos Sociales del Gobierno de Canarias.

GARCÍA BORREGO, I. (2003). Los hijos de inmigrantes como tema sociológico: la cuestión de "la segunda generación, Anduli: revista andaluza de ciencias sociales, 3, pp. 27-46.

GODENAU, D. y BURASCHI, D. (coords.) (2017). Migraciones, desigualdad e integración en tiempos de crisis. Cabildo Insular de Tenerife. Área de Empleo, Comercio, Industria y Desarrollo Económico.

GOFFMAN, E. (2006). Estigma. La identidad deteriorada. Buenos Aires, Amorrortu. 
HEATH, A. (2014) "Introduction: Patterns of generational change: convergent, reactive or emergent?", Ethnic and Racial Studies, 37(1), pp. 1-9.

KHAN, S. A. (2011) "Location Nowhere: Alienation, Nihilism and Radicalism among British Pakistani Muslim Youth". En: David, M. y Muñoz-Basols, J. eds. Defining and Re-defining Diaspora. From Theory to Reality. Oxford: Inter-Disciplinary Press.

KUEHN DUMPIÉRREZ, R. (2007). ¿Jóvenes o inmigrantes? Inmigración, juventudes y planificación social en Canarias. En Cachón Rodríguez, L. y López Sala, A. (Coords.) (2007). Juventud e Inmigración, Desafíos para la Participación y para la Integración, pp. 64-90. Dirección General de Juventud de la Consejería de Empleo y Asuntos Sociales del Gobierno de Canarias.

LAMONT, M. y MOLNÁR, V. (2002) "The Study of Boundaries in the Social Sciences", Annual Review of Sociology, 28, pp. 167-195.

PARK, R. E. (1930) "Assimilation, Social". En: Seligman, E. y Johnson, A. (eds). Encyclopedia of the Social Sciences.

PORTES, A., APARICIO, R. y HALLER, W. (2009). La segunda generación en Madrid: un estudio longitudinal. Real Instituto Elcano, ARI 67/2009.

PORTES, A. y APARICIO, R. (2013). Investigación longitudinal sobre la segunda generación en España: reporte de avance. ARI 34/2013. Real Instituto Elcano.

PORTES, A., APARICIO, R. y HALLER, W. (2018). Hacerse adulto en España. La integración de los hijos de inmigrantes. Anuario CIDOB de la Inmigración 2018, pp. 148-181.

PORTES, A. y RUMBAUT, R. (2001). Legacies. The Story of the Immigrant Second Generation, California University Press.

PORTES, A. y ZHOU, M. (1993). The New Second Generation and Its Variants, Annals of the American Academy of Political and social Science, 530, pp. 74-96.

PORTES, A., VICKSTROM, E. y APARICIO, R. (2013). Hacerse adulto en España. Autoidentificación, creencias y autoestima de los hijos de inmigrantes. Papers, 98(2), pp. 148-181.

RAMÍREZ, A. y MIJARES, L. (2005). Gestión del Islam y de la Inmigración en Europa: Tres estudios de caso, Migraciones, 18, pp. 77-104.

RUMBAUT, R.G. (2008). "Reaping What you Sow: Immigration, Youth, and Reactive Ethnicity", Applied Developmental Science, 12(2), pp. 108-111.

SARABIA MEDEL, J. (Dir.) (2007). Evaluación e investigación educativa. Inmigración, escolarización y Educación Intercultural en Canarias. Características sociales y académicas del alumnado inmigrante de Educación Infantil, Primaria y Secundaria Obligatoria. Consejería de Educación, Cultura y Deportes del Gobierno de Canarias.

TÖLÖLYAN, K. (2007). "The Contemporary Discourse of Diaspora Studies", Comparative Studies of South Asia, Africa and the Middle East, 27(3), pp. 647-655. 


\section{Cómo citar este artículo:}

CABRERA ABU, N. (2020). Las Segundas Generaciones de Origen Extranjero. OBITen Factsheet 9-2020. Recuperado de www.obiten.net. DOI: https://doi.org/10.25145/r.obitfact.2020.03.

El Observatorio de la Inmigración de Tenerife es una iniciativa conjunta del Cabildo de Tenerife y la Universidad de La Laguna que surge con vocación de estructura permanente y dinámica para avanzar en el conocimiento científico de los movimientos migratorios. OBITen desarrolla su actividad a modo de centro de recopilación, producción y difusión de información que facilita la opinión cualificada y favorece la toma de decisiones que redunden en una mejor gestión del fenómeno inmigratorio y sus implicaciones.

\section{http://www.obiten.net}

